\title{
REPRESENTATION FUNCTIONS OF ADDITIVE BASES FOR ABELIAN SEMIGROUPS
}

\author{
MELVYN B. NATHANSON
}

Received 3 June 2003

\begin{abstract}
A subset of an abelian semigroup is called an asymptotic basis for the semigroup if every element of the semigroup with at most finitely many exceptions can be represented as the sum of two distinct elements of the basis. The representation function of the basis counts the number of representations of an element of the semigroup as the sum of two distinct elements of the basis. Suppose there is given function from the semigroup into the set of nonnegative integers together with infinity such that this function has only finitely many zeros. It is proved that for a large class of countably infinite abelian semigroups, there exists a basis whose representation function is exactly equal to the given function for every element in the semigroup.
\end{abstract}

2000 Mathematics Subject Classification: 11B13, 11B34, $11 \mathrm{~B} 05$.

1. Additive bases for semigroups. Let $\mathbb{N}, \mathbb{N}_{0}$, and $\mathbb{Z}$ denote the positive integers, nonnegative integers, and integers, respectively. Let $X$ be an abelian semigroup, written additively, and let $A$ and $B$ be subsets of $X$. We define the sumset

$$
A+B=\{a+b: a \in A, b \in B\}
$$

and the restricted sumset

$$
A \hat{+} B=\{a+b: a \in A, b \in B, a \neq b\} .
$$

In particular, for $A=B$, we have

$$
\begin{gathered}
2 B=B+B=\left\{b+b^{\prime}: b, b^{\prime} \in B\right\}, \\
2 \wedge B=B \hat{+} B=\left\{b+b^{\prime}: b, b^{\prime} \in B, b \neq b^{\prime}\right\} .
\end{gathered}
$$

For every positive integer $h$ we introduce the dilation

$$
h * B=\{h b: b \in B\}=\{\underbrace{b+\cdots+b}_{h \text { summands }}: b \in B\} .
$$

Then,

$$
2 B=(2 \wedge B) \cup(2 * B) .
$$

Additive number theory is classically the study of sums of subsets of the semigroup of nonnegative integers. In this paper, we extend the classical theory to a large class of abelian semigroups. 
If $X$ is a group and $A, B \subseteq X$, then we can also define the difference set

$$
A-B=\{a-b: a \in A, b \in B\} .
$$

We have $-A=\{0\}-A=\{-a: a \in A\}$.

Let $B$ be a subset of the semigroup $X$. We associate to $B$ two representation functions, $\hat{r}_{B}(x)$ and $r_{B}(x)$. The restricted representation function

$$
\hat{r}_{B}: X \rightarrow \mathbb{N}_{0} \cup\{\infty\}
$$

of the set $B$ counts the number of ways an element $x \in X$ can be written as a sum of two distinct elements of $B$, that is,

$$
\hat{r}_{B}(x)=\operatorname{card}\left(\left\{b, b^{\prime}\right\} \subseteq B: b+b^{\prime}=x, b \neq b^{\prime}\right) .
$$

The representation function

$$
r_{B}: X \rightarrow \mathbb{N}_{0} \cup\{\infty\}
$$

of the set $B$ counts the number of ways an element $x \in X$ can be written as a sum of two not necessarily distinct elements of $B$, that is,

$$
r_{B}(x)=\operatorname{card}\left(\left\{b, b^{\prime}\right\} \subseteq B: b+b^{\prime}=x\right) .
$$

If every element of $X$ can be represented as the sum of two distinct elements of $B$, that is, if $2 \wedge B=X$, or, equivalently, if $\hat{r}_{B}(x) \geq 1$ for all $x \in X$, then the set $B$ is called a restricted basis for $X$. If $\hat{r}_{B}(x) \geq 1$ for all but finitely many $x \in X$, then $B$ is called a restricted asymptotic basis for $X$.

Similarly, if every element of $X$ can be represented as the sum of two not necessarily distinct elements of $B$, that is, if $2 B=X$, or, equivalently, if $r_{B}(x) \geq 1$ for all $x \in X$, then the set $B$ is called a basis for $X$. If $r_{B}(x) \geq 1$ for all but finitely many $x \in X$, then the set $B$ is called an asymptotic basis for $X$.

A famous conjecture of Erdős and Turán [2] in additive number theory states that if a set $B$ of nonnegative integers is an asymptotic basis or a restricted asymptotic basis for $\mathbb{N}_{0}$, then the representation functions $r_{B}(x)$ and $\hat{r}_{B}(x)$ must be unbounded. This is still an unsolved problem for the semigroup of nonnegative integers under addition, but analogues of the Erdős-Turán conjecture do hold in some other abelian semigroups. For example, let $a$ and $b$ be nonnegative integers and define $a * b=\max (a, b)$. Then $\left(\mathbb{N}_{0}, *\right)$ is an abelian semigroup with identity 0 . If $B$ is a nonempty subset of $\mathbb{N}_{0}$, then $2 B=B$ and $2 \wedge B=B \backslash\{\min (B)\}$ in $\left(\mathbb{N}_{0}, *\right)$. It follows that if $B$ is an asymptotic basis or a restricted asymptotic basis for $\mathbb{N}_{0}$, then $B$ must contain all but finitely many nonnegative integers. Moreover, if $\operatorname{card}\left(\mathbb{N}_{0} \backslash B\right)=t$, then $r_{B}(n)=n+1-t$ and $\hat{r}_{B}(n)=n-t$ for all sufficiently large $n$, and so

$$
\limsup _{n \rightarrow \infty} r_{B}(n)=\limsup _{n \rightarrow \infty} \hat{r}_{B}(n)=\infty .
$$

For the semigroup $(\mathbb{N}, \cdot)$ of positive integers under ordinary multiplication, Erdős [1] proved that if $B$ is an asymptotic basis for the multiplicative semigroup $\mathbb{N}$, then 
the representation function $r_{B}(n)$ is unbounded. Nešetřil and Rödl [6] gave a simple, Ramsey-theoretic proof of this result, and Nathanson [4] and Puš [8] generalized Erdós' theorem in different directions.

The story is very different for the abelian group $\mathbb{Z}$ of integers. There exist subsets $B$ of integers such that $2 B=\mathbb{Z}$ and the representation function $r_{B}(x)$ is bounded. Indeed, Nathanson [5] has constructed "dense" bases $B$ for the integers such that $r_{B}(x)=1$ for all $x \in \mathbb{Z}$. Similarly, there exist subsets $B$ of integers such that $2 \wedge B=\mathbb{Z}$ and $\hat{r}_{B}(x)=1$ for all $x \in \mathbb{Z}$. A special case of a theorem in this paper is that there is no constraint on the restricted representation functions of restricted asymptotic bases for the integers, nor on the representation functions of asymptotic bases for the integers. This means that for any function

$$
f: \mathbb{Z} \longrightarrow \mathbb{N}_{0} \cup\{\infty\}
$$

such that

$$
\operatorname{card}\left(f^{-1}(0)\right)<\infty \text {, }
$$

there exists a set $B \subseteq \mathbb{Z}$ such that $\hat{r}_{B}(x)=f(x)$ for all $x \in \mathbb{Z}$. Indeed, we prove that if $G$ is any countable abelian group such that $2 * G$ is infinite, then every function

$$
f: G \longrightarrow \mathbb{N}_{0} \cup\{\infty\}
$$

with $\operatorname{card}\left(f^{-1}(0)\right)<\infty$, is the restricted representation function $\hat{r}_{B}$ of some set $B \subseteq G$, and is also the representation function $\hat{r}_{B^{\prime}}$ of some set $B^{\prime} \subseteq G$.

In this paper, we study the more general case of additive abelian semigroups of the form $X=S \oplus G$, where $S$ is a countable abelian semigroup and $G$ is a countably infinite abelian group. A semigroup of the form $S \oplus G$, where $S$ is a semigroup and $G$ is a group, will be called a semigroup with a group component. The main result of this paper states that if $X=S \oplus G$ is an abelian semigroup with a group component $G$ such that $S+S=S$ and $2 * G$ is infinite, then essentially every function is the representation function of a restricted asymptotic basis for $X$. A special case of this theorem for groups was obtained by Puš [7].

2. Representation functions of semigroups with a group component. Let $S$ be a countable abelian semigroup, and let $G$ be a countably infinite abelian group. Both $S$ and $G$ are written additively. We do not assume that $S$ is infinite. We will describe the representation functions of asymptotic bases and restricted asymptotic bases for the additive abelian semigroup

$$
X=S \oplus G=\{(s, g): s \in S \text { and } g \in G\}
$$

For $x=(s, g) \in X$, we have the projection map $\pi: X \rightarrow G$ defined by $\pi(x)=g$.

We begin with a simple lemma about abelian groups with finite dilation. 
LEMMA 2.1. Let $h \geq 2$ and let $G$ be a countably infinite abelian group. The dilation $h * G$ is finite if and only if

$$
G \cong G_{0} \oplus\left(\bigoplus_{\substack{d \mid h \\ d \geq 2}} G_{d}\right),
$$

where $G_{0}$ is a finite abelian group and $G_{d}$ is a direct sum of cyclic groups of order $d$.

Proof. Let $G$ be a group of the form (2.2). If the positive integer $d$ divides $h$ and if $\Gamma_{d}$ is a cyclic group of order $d$, then $h * \Gamma_{d}=\{0\}$ and so $h * G_{d}=\{0\}$. It follows that $h * G=h * G_{0}$ is finite.

Conversely, suppose that $h * G$ is finite. Let $\gamma \in G$. For $k \geq 1$ we have $h^{k} \gamma=h\left(h^{k-1} \gamma\right)$, and so

$$
\left\{h^{k} \gamma: k=1,2,3, \ldots\right\} \subseteq h * G .
$$

Since $h * G$ is finite, there exist positive integers $j<k$ such that $h^{j} \gamma=h^{k} \gamma$, and so $\gamma$ has finite order. Therefore, $G$ is a torsion group.

Let $m$ be the least common multiple of the orders of the elements of the finite set $h * G$. Then, $(m h) \gamma=m(h \gamma)=0$ for all $\gamma \in G$, and so $G$ is a group of bounded order. Since an abelian group of bounded order is a direct sum of nonzero cyclic groups [3, Theorem 6], we can write

$$
G=\bigoplus_{i=1}^{\infty} \Gamma_{d_{i}}
$$

where $\Gamma_{d}$ denotes the cyclic group of order $d$. Let $\gamma_{i}$ be a generator of the group $\Gamma_{d_{i}}$. Since $h * G$ is finite, it follows that $h \gamma_{i}=0$ for all but finitely many $i$. The set

$$
I_{0}=\left\{i \geq 1: h \gamma_{i} \neq 0\right\}
$$

is finite, and

$$
G_{0}=\bigoplus_{i \in I_{0}} \Gamma_{d_{i}}
$$

is a finite abelian group. If $h \gamma_{i}=0$, then $\gamma_{i}$ has order $d$ for some divisor $d$ of $h, d \geq 2$. For every divisor $d$ of $h$ with $d \geq 2$, we define

$$
\begin{gathered}
I_{d}=\left\{i \geq 1: \gamma_{i} \text { has order } d\right\}, \\
G_{d}=\bigoplus_{i \in I_{d}} \Gamma_{d} .
\end{gathered}
$$

The set $\bigcup_{d \mid h} I_{d}$ is infinite, and (2.2) holds. This completes the proof.

Let $\Gamma_{2}^{\infty}=\bigoplus_{i=1}^{\infty} \Gamma_{2}$ denote the direct sum of an infinite number of cyclic groups of order 2. 
LEMMA 2.2. Let $G$ be a countably infinite abelian group. Then $2 * G$ is finite if and only if

$$
G \cong G_{0} \oplus \Gamma_{2}^{\infty}
$$

for some finite abelian group $G_{0}$.

Proof. This is the special case of Lemma 2.1 for $h=2$.

We consider semigroups $S$ with the property that $S+S=S$. Equivalently, for every $s \in S$ there exist $s^{\prime}, s^{\prime \prime} \in S$ such that $s=s^{\prime}+s^{\prime \prime}$. Every semigroup with identity has this property, since $s=s+0$. If $S$ is any totally ordered set without a smallest element, and if we define $s_{1}+s_{2}=\max \left(s_{1}, s_{2}\right)$, then $S$ is an abelian semigroup such that $s=s+s$ for all $s \in S$, but $S$ does not have an identity element.

THEOREM 2.3. Let $S$ be a countable abelian semigroup such that for every $\in \in S$ there exist $s^{\prime}, s^{\prime \prime} \in S$ with $s=s^{\prime}+s^{\prime \prime}$. Let $G$ be a countably infinite abelian group such that the dilation $2 * G$ is infinite. Consider the abelian semigroup $X=S \oplus G$ with projection map $\pi: X \rightarrow$ G. Let

$$
f: X \rightarrow \mathbb{N}_{0} \cup\{\infty\}
$$

be any map such that the set

$$
Z_{0}=\pi\left(f^{-1}(0)\right)
$$

is a finite subset of $G$. Then, there exists a set $B \subseteq X$ such that

$$
\hat{r}_{B}(x)=f(x)
$$

for all $x \in X$, where $\hat{r}_{B}(x)$ denotes the number of sets $\left\{b, b^{\prime}\right\} \subseteq B$, such that $b \neq b^{\prime}$ and $b+b^{\prime}=x$.

Note that Theorem 2.3 is not true for all abelian semigroups. For example, let $\mathbb{N}$ be the additive semigroup of positive integers under addition, and $X=\mathbb{N} \oplus \mathbb{Z}$. For every set $B \subset X$ we have $\hat{r}_{B}(1, n)=r_{B}(1, n)=0$ for every $n \in \mathbb{Z}$.

Proof. We will construct inductively an increasing sequence $B_{1} \subseteq B_{2} \subseteq \cdots$ of finite subsets of $X$ such that the set $B=\bigcup_{n=1}^{\infty} B_{n}$ has the property that $\hat{r}_{B}(x)=f(x)$ for all $x \in X$.

Since the dilation $2 * G$ is infinite and the set $Z_{0}=\pi\left(f^{-1}(0)\right)$ is finite, we can choose an infinite subset $U$ of $G$ such that $U \cap Z_{0}=\varnothing$, and if $u, u^{\prime} \in U$ and $u \neq u^{\prime}$, then $2 u \neq 2 u^{\prime}$.

Let $\left\{x_{i}\right\}_{i=1}^{\infty}$ be a sequence of elements of $X$ such that, for every $x \in X$,

$$
f(x)=\operatorname{card}\left\{i \in \mathbb{N}: x_{i}=x\right\} .
$$


Let $x_{1}=\left(s_{1}, g_{1}\right) \in S \oplus G=X$. Then, $f\left(x_{1}\right) \geq 1$. Since $U$ is infinite, we can choose $u_{1} \in U$ such that

$$
2 u_{1} \neq g_{1} .
$$

Choose $s_{1}^{\prime}, s_{1}^{\prime \prime} \in S$ such that $s_{1}=s_{1}^{\prime}+s_{2}^{\prime \prime}$. We define

$$
B_{1}=\left\{\left(s_{1}^{\prime}, g_{1}-u_{1}\right),\left(s_{1}^{\prime \prime}, u_{1}\right)\right\} .
$$

Then, $\left(s_{1}^{\prime}, g_{1}-u_{1}\right) \neq\left(s_{1}^{\prime \prime}, u_{1}\right)$ and

$$
2 \wedge B_{1}=\left\{\left(s_{1}, g_{1}\right)\right\}=\left\{x_{1}\right\} .
$$

Then,

$$
\hat{r}_{B_{1}}(x)= \begin{cases}1 & \text { if } x=x_{1} \\ 0 & \text { otherwise }\end{cases}
$$

and so

$$
\hat{r}_{B_{1}}(x) \leq f(x) \quad \forall x \in X
$$

Let $n \geq 2$, and suppose that we have constructed finite sets

$$
B_{1} \subseteq B_{2} \subseteq \cdots \subseteq B_{n-1} \subseteq X
$$

such that

$$
\hat{r}_{B_{n-1}}(x) \leq f(x) \quad \forall x \in X,
$$

and, for $i=1,2, \ldots, n-1$,

$$
\hat{r}_{B_{n-1}}\left(x_{i}\right) \geq \operatorname{card}\left\{j \leq n-1: x_{j}=x_{i}\right\} .
$$

Let $x_{n}=\left(s_{n}, g_{n}\right) \in X$. If $\hat{r}_{B_{n-1}}\left(x_{n}\right)=f\left(x_{n}\right)$, then we set $B_{n}=B_{n-1}$. Suppose that $\hat{r}_{B_{n-1}}\left(x_{n}\right)<f\left(x_{n}\right)$. Since $U$ is an infinite subset of the group $G$, and $\pi\left(B_{n-1}\right)$ and $\pi(2 \wedge$ $\left.B_{n-1}\right)$ are finite subsets of $G$, we can choose an element $u_{n} \in U$ satisfying the following conditions:

(i) $2 u_{n} \neq g_{n}$,

(ii) $u_{n} \notin \pi\left(B_{n-1}\right) \cup\left(\left\{g_{n}\right\}-\pi\left(B_{n-1}\right)\right)$,

(iii) $u_{n} \notin \pi\left(B_{n-1}\right)+\left\{g_{n}\right\}-\pi\left(2 \wedge B_{n-1}\right)$,

(iv) $u_{n} \notin \pi\left(B_{n-1}\right)+\left\{g_{n}\right\}-Z_{0}$,

(v) $u_{n} \notin \pi\left(2 \wedge B_{n-1}\right)-\pi\left(B_{n-1}\right)$,

(vi) $u_{n} \notin Z_{0}-\pi\left(B_{n-1}\right)$,

(vii) $2 u_{n} \notin \pi\left(B_{n-1}\right)+\left\{g_{n}\right\}-\pi\left(B_{n-1}\right)$.

Choose $s_{n}^{\prime}, s_{n}^{\prime \prime} \in S$ such that $s_{n}=s_{n}^{\prime}+s_{n}^{\prime \prime}$, and let

$$
B_{n}=B_{n-1} \cup\left\{\left(s_{n}^{\prime}, g_{n}-u_{n}\right),\left(s_{n}^{\prime \prime}, u_{n}\right)\right\} .
$$


It follows from (i) and (ii) that

$$
\begin{gathered}
\left(s_{n}^{\prime}, g_{n}-u_{n}\right) \neq\left(s_{n}^{\prime \prime}, u_{n}\right), \\
B_{n-1} \cap\left\{\left(s_{n}^{\prime}, g_{n}-u_{n}\right),\left(s_{n}^{\prime \prime}, u_{n}\right)\right\}=\varnothing .
\end{gathered}
$$

Therefore,

$$
2 \wedge B_{n}=2 \wedge B_{n-1} \cup\left(B_{n-1}+\left\{\left(s_{n}^{\prime}, g_{n}-u_{n}\right),\left(s_{n}^{\prime \prime}, u_{n}\right)\right\}\right) \cup\left\{x_{n}\right\} .
$$

It follows from (iii) and (iv) that

$$
\left(B_{n-1}+\left\{\left(s_{n}^{\prime}, g_{n}-u_{n}\right)\right\}\right) \cap 2 \wedge B_{n-1}=\varnothing
$$

and that

$$
f(x) \geq 1 \quad \forall x \in B_{n-1}+\left\{\left(s_{n}^{\prime}, g_{n}-u_{n}\right)\right\} .
$$

Similarly, (v) and (vi) imply that

$$
\left(B_{n-1}+\left\{\left(s_{n}^{\prime \prime}, u_{n}\right)\right\}\right) \cap 2 \wedge B_{n-1}=\varnothing
$$

and that

$$
f(x) \geq 1 \quad \forall x \in B_{n-1}+\left\{\left(s_{n}^{\prime \prime}, u_{n}\right)\right\}
$$

It follows from (vii) that

$$
\left(B_{n-1}+\left\{\left(s_{n}^{\prime}, g_{n}-u_{n}\right)\right\}\right) \cap\left(B_{n-1}+\left\{\left(s_{n}^{\prime \prime}, u_{n}\right)\right\}\right)=\varnothing .
$$

Conditions (ii) and (iii) imply that

$$
x_{n} \notin B_{n-1}+\left\{\left(s_{n}^{\prime}, g_{n}-u_{n}\right),\left(s_{n}^{\prime \prime}, u_{n}\right)\right\} .
$$

Therefore,

$$
\begin{gathered}
\hat{r}_{B_{n}}\left(x_{n}\right)=\hat{r}_{B_{n-1}}\left(x_{n}\right)+1 \geq \operatorname{card}\left\{j \leq n: x_{j}=x_{n}\right\}, \\
\hat{r}_{B_{n}}(x)= \begin{cases}\hat{r}_{B_{n-1}}(x) & \text { for } x \in\left(2 \wedge B_{n-1}\right) \backslash\left\{x_{n}\right\}, \\
1 & \text { for } x \in B_{n-1}+\left\{\left(s_{n}^{\prime}, g_{n}-u_{n}\right),\left(s_{n}^{\prime \prime}, u_{n}\right)\right\},\end{cases}
\end{gathered}
$$

hence $\hat{r}_{B_{n}}(x) \leq f(x)$ for all $x \in X$.

This construction produces a sequence $\left\{B_{n}\right\}_{n=1}^{\infty}$ of finite sets with the property that the infinite set $B=\bigcup_{n=1}^{\infty} B_{n}$ satisfies $\hat{r}_{B}(x)=f(x)$ for all $x \in X$. This completes the proof. 
THEOREM 2.4. Let $G$ be a countably infinite abelian group such that the dilation $2 * G$ is infinite. Let $f: G \rightarrow \mathbb{N}_{0} \cup\{\infty\}$ be any map such that the set

$$
Z_{0}=f^{-1}(0)
$$

is a finite subset of $G$. Then there exists a restricted asymptotic basis $B$ of order 2 for $G$ such that $\hat{r}_{B}(x)=f(x)$ for all $x \in X$, where $\hat{r}_{B}(x)$ denotes the number of sets $\left\{b, b^{\prime}\right\} \subseteq B$ such that $b \neq b^{\prime}$ and $b+b^{\prime}=x$.

Proof. This follows immediately from Theorem 2.3 with $S=\{0\}$.

By the same methods we can prove the following theorems about unrestricted representation functions. In the unrestricted case, there are additional conditions that must be satisfied for the proofs to work. It suffices to replace $2 * G$ by $12 * G$ in the statements of Theorems 2.5 and 2.6.

THEOREM 2.5. Let $S$ be a countable abelian semigroup, such that for everys $S \in S$ there exist $s^{\prime}, s^{\prime \prime} \in S$ with $s=s^{\prime}+s^{\prime \prime}$. Let $G$ be a countably infinite abelian group such that the dilation $12 * G$ is infinite. Consider the abelian semigroup $X=S \oplus G$ with projection map $\pi: X \rightarrow G$. Let $f: X \rightarrow \mathbb{N}_{0} \cup\{\infty\}$ be any map such that the set $Z_{0}=f^{-1}(0)$ is finite. Then, there exists a set $B \subseteq X$ such that

$$
r_{B}(x)=f(x)
$$

for all $x \in X$, where $r_{B}(x)$ denotes the number of sets $\left\{b, b^{\prime}\right\} \subseteq B$ such that $b+b^{\prime}=x$.

THEOREM 2.6. Let $G$ be a countably infinite abelian group such that the dilation $12 * G$ is infinite. Let $f: G \rightarrow \mathbb{N}_{0} \cup\{\infty\}$ be any map such that the set $Z_{0}=f^{-1}(0)$ is finite. Then, there exists an asymptotic basis $B$ of order 2 for $G$ such that $r_{B}(x)=f(x)$ for all $x \in X$, where $r_{B}(x)$ denotes the number of sets $\left\{b, b^{\prime}\right\} \subseteq B$ such that $b+b^{\prime}=x$.

3. Bases for groups of exponent 2. Let $X=S \oplus G$ be an abelian semigroup with group component $G$. If $2 * G$ is finite, then it is an open problem to classify the representation functions of asymptotic bases and restricted asymptotic bases for $X$. We know from Lemma 2.2 that $G \cong G_{0} \oplus \Gamma_{2}^{\infty}$, where $G_{0}$ is a finite abelian group. Replacing the semigroup $S$ with $S \oplus G_{0}$, we see that it suffices to consider semigroups of the form $X=S \oplus \Gamma_{2}^{\infty}$. Even the special case $S=\{0\}$ and $X=\Gamma_{2}^{\infty}$ is a mystery. If $x, x^{\prime} \in \Gamma_{2}^{\infty}$, then $x+x^{\prime}=0$ if and only if $x=x^{\prime}$, and so, for any subset $B$ of $\Gamma_{2}^{\infty}$, we have $\hat{r}_{B}(0)=0, r_{B}(0)=\operatorname{card}(B)$, and $\hat{r}_{B}(x)=r_{B}(x)$ for all $x \neq 0$.

The following result shows another constraint on the representation functions of asymptotic bases for $\Gamma_{2}^{\infty}$.

LEMmA 3.1. Let $G=\Gamma_{2}^{\infty}$ be an infinite direct sum of cyclic groups of order 2. Let $B$ be a subset of $G$, and let $\hat{r}_{B}(x)$ be the restricted representation function of $B$. If $\hat{r}_{B}(x) \geq 2$ for some $x \in G$, then there exist elements $y, z \in G$ such that $\hat{r}_{B}(y) \geq 2$ and $\hat{r}_{B}(z) \geq 2$, and the elements $x, y$, and $z$ are distinct. 
Proof. If $\hat{r}_{B}(x) \geq 2$, then there exist distinct elements $a, b, c, d \in G$ such that

$$
x=a+b=c+d .
$$

Since every element of $G$ has order 2, we have

$$
\begin{aligned}
& y=a+c=a-c=d-b=b+d, \\
& z=a+d=a-d=c-b=b+c,
\end{aligned}
$$

and so $\hat{r}_{B}(y) \geq 2$ and $\hat{r}_{B}(z) \geq 2$. The elements $x, y$, and $z$ are distinct. This completes the proof.

THEOREM 3.2. Let $G=\Gamma_{2}^{\infty}$, and let $f: G \rightarrow \mathbb{N}_{0} \cup \infty$ be a function, such that $f(x) \geq 1$ for all $x \in G$, and $f(x) \geq 2$ for exactly one or two elements of $G$. There does not exist a set $B$ in $G$ such that $\hat{r}_{B}(x)=f(x)$.

Proof. This follows immediately from Lemma 3.1.

It is not hard to construct a basis $B$ for $\Gamma_{2}^{\infty}$ such that $r_{B}(x)=1$ for all $x \neq 0$, but it is an open problem to describe all representation functions for this group.

ACKNOWLEDGMENT. This work was supported in part by grants from the National Security Agency (NSA) Mathematical Sciences Program and the Professional Staff Congress-City University of New York (PSC-CUNY) Research Award Program.

\section{REFERENCES}

[1] P. Erdôs, On the multiplicative representation of integers, Israel J. Math. 2 (1964), 251-261.

[2] P. Erdős and P. Turán, On a problem of Sidon in additive number theory, and on some related problems, J. London Math. Soc. 16 (1941), 212-215.

[3] I. Kaplansky, Infinite Abelian Groups, University of Michigan Press, Ann Arbor, 1954.

[4] M. B. Nathanson, Multiplicative representations of integers, Israel J. Math. 57 (1987), no. 2, 129-136.

[5] __ Unique representation bases for the integers, Acta Arith. 108 (2003), no. 1, 1-8.

[6] J. Nešetřil and V. Rödl, Two proofs in combinatorial number theory, Proc. Amer. Math. Soc. 93 (1985), no. 1, 185-188.

[7] V. Puš, On multiplicative bases in abelian groups, Czechoslovak Math. J. 41(116) (1991), no. 2, 282-287.

[8] _ On multiplicative bases in commutative semigroups, European J. Combin. 13 (1992), no. 3, 201-211.

Melvyn B. Nathanson: Department of Mathematics, Lehman College, City University of New York (CUNY), Bronx, NY 10468, USA

E-mail address: nathansn@a1pha. 1 ehman. cuny. edu 


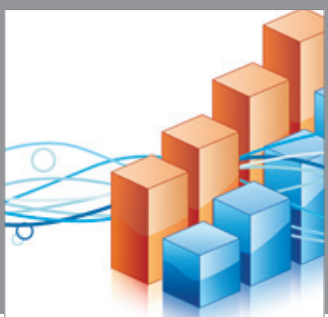

Advances in

Operations Research

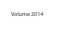

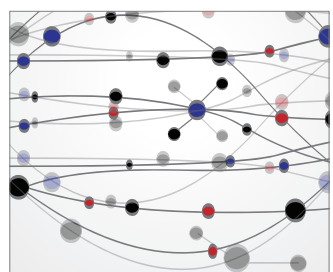

\section{The Scientific} World Journal


International Journal of

Mathematics and

Mathematical

Sciences
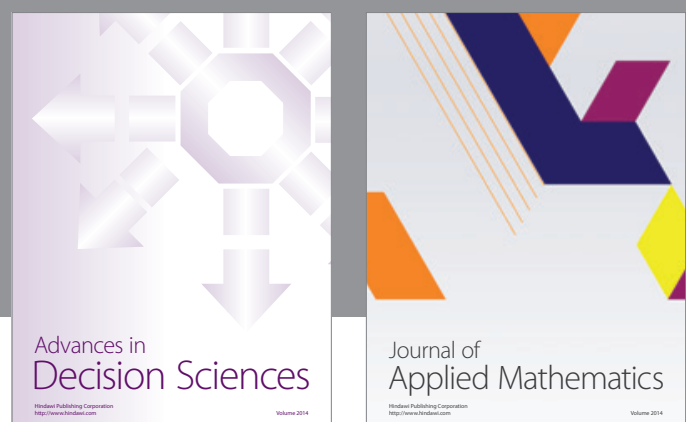

Journal of

Applied Mathematics
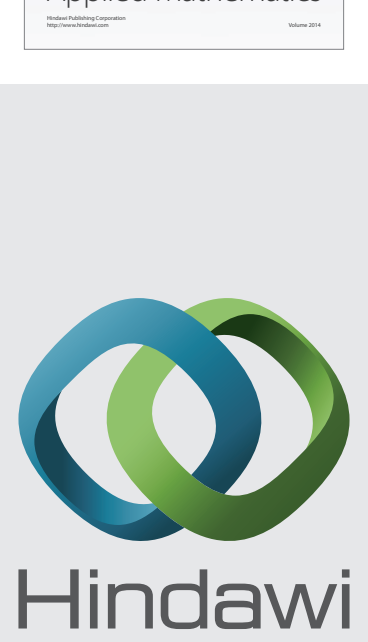

Submit your manuscripts at http://www.hindawi.com
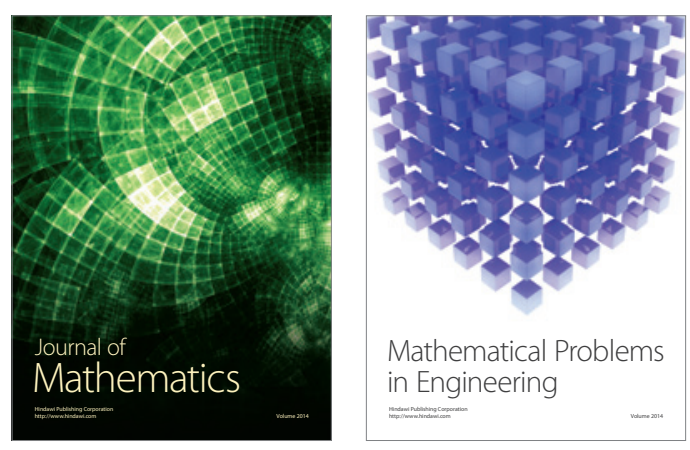

Mathematical Problems in Engineering
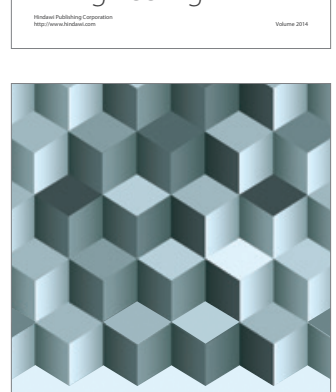

Journal of

Function Spaces
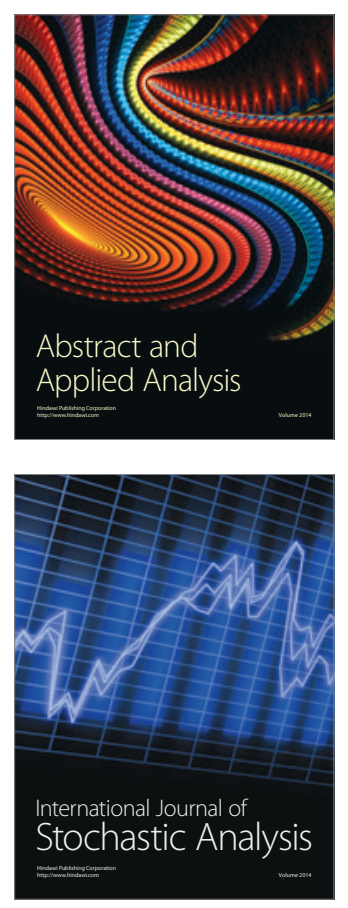

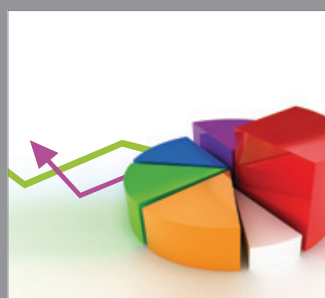

ournal of

Probability and Statistics

Promensencen
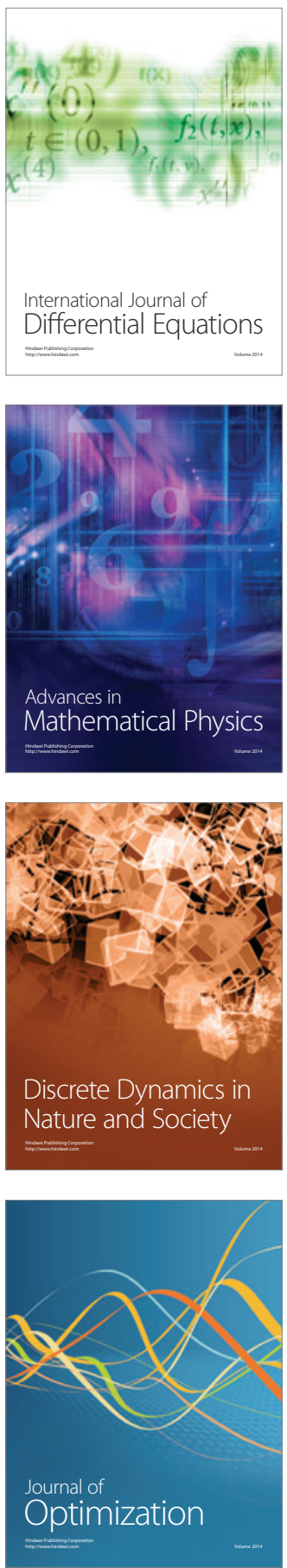